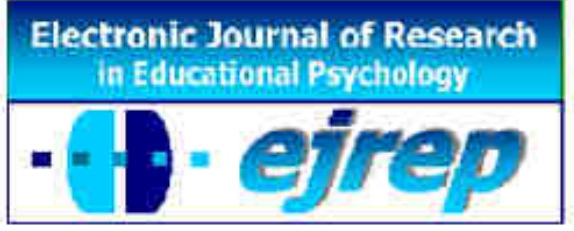

\title{
La invención de problemas aritméticos por es- tudiantes con talento matemático
}

\author{
Johan Espinoza González ${ }^{1}$ José Luis Lupiáñez Gómez ${ }^{2}$ \\ e Isidoro Segovia Alex ${ }^{2}$ \\ ${ }^{1}$ Universidad Nacional de Costa Rica \\ ${ }^{2}$ Departamento de Didáctica de la Matemática, Universidad de Granada.
}

\section{Costa Rica / España}

Correspondencia: Johan Espinoza González. Universidad Nacional, Sede Regional Brunca. Código postal: 348000. San Isidro del General, Pérez Zeledón, Costa Rica. E-mail: jespinoza@una.cr

(C) Education \& Psychology I+D+i and Ilustre Colegio Oficial de la Psicología de Andalucía Oriental (Spain) 


\section{Resumen}

Introducción. Se presenta el análisis de los problemas aritméticos planteados por un grupo de estudiantes considerados con talento matemático ante dos tareas de invención de problemas y su comparación con las actuaciones que presentan un grupo estándar de estudiantes de un colegio público ante las mismas tareas. Este estudio se centró en caracterizar e identificar las diferencias entre las producciones de ambos grupos, con base en un esquema de análisis confeccionado en esta investigación.

Método. La muestra está conformada por dos grupos de estudiantes. El primero está compuesto por 21 estudiantes identificados con talento matemático y el segundo está formado por 19 estudiantes de segundo curso de Educación Secundaria de un colegio público. Ambos grupos contestaron dos tareas de invención de problemas aritméticos que fueron confeccionadas en este estudio. Los resultados se analizaron con base en tres categorías de análisis y algunas variables que están relacionadas con la estructura sintáctica, semántica y matemática de los mismos.

Resultados. Los problemas inventados por el grupo de estudiantes con talento en matemática presentan mayor riqueza que los del grupo del colegio público, ya que están conformados por una mayor cantidad de proposiciones, emplean diferentes tipos de números, requieren más pasos y procesos de cálculo distintos para ser resueltos y presentan una mayor cantidad de relaciones semánticas distintas. Además, los resultados muestran algunos atributos que caracterizan a los estudiantes con talento en matemática cuando resuelven tareas de invención de problemas.

Conclusión. El instrumento de invención de problemas y el esquema analítico empleado permitieron describir y explorar la actuación de un grupo de estudiantes considerados con talento matemático. Además, las tres categorías de análisis y la solución de cada problema dan lugar a diez componentes cuyos valores caracterizan la riqueza de los problemas inventados por los sujetos. Por último, el análisis estadístico realizado refuerza las diferencias encontradas entre las producciones de ambos grupos en estudio.

Palabras Clave: Talento matemático, invención de problemas, riqueza de enunciados, problemas aritméticos, Educación Matemática. 


\title{
The posing of arithmetic problems by mathematically talented students
}

\begin{abstract}
Introduction. The analysis of the arithmetic problems posed by a group of mathematically talented students considered at two tasks of inventing problems and their comparison with the actions that have a standard group of students from a public school to the same tasks is presented. This study focused on characterizing and identifying the differences between the productions of both groups, based on a framework of analysis made in this study.
\end{abstract}

Method. The sample consists of two groups of students. The first one consists of 21 students identified talented in math and the second one consists of 20 secondary students from a public school. Both groups completed two tasks of invention of arithmetic problems that were not made in this study. The results were analyzed based on three categories of analysis and some variables that are related to the syntactic, semantic and mathematical structure thereof.

Results. The problems invented by a group of talented students in mathematics have greater value than the group of public school because they are composed of a greater number of propositions, use different types of numbers, require more steps and processes different calculation to be solved and have a greater number of different semantic relationships. Furthermore, the results show some attributes that characterize talented students in mathematics when solving tasks of inventing problems.

Conclusion. The instrument of inventing problems and the analytical framework used to describe and explore allowed the performance of a group of students considered with mathematical talent. Moreover, the three categories of analysis employed and the solvability of every problem lead to ten components whose values characterize the wealth of problems invented by the subjects. Finally, statistical analysis reinforces the differences between the productions of both groups in the study.

Keywords: Mathematical talent, problem posing, richness of statements, arithmetic problems, Mathematics Education 


\section{Introducción}

Esta investigación se centró en caracterizar las producciones en invención de problemas aritméticos de un grupo de sujetos identificados con talento matemático y compararlas con las que realiza un grupo estándar. Así, el problema de investigación considerado en este trabajo comprende dos campos de estudio: la invención de problemas y los sujetos con talento matemático. Con respecto a la invención de problemas, ésta es una línea de investigación que surge de estudios realizados en resolución de problemas (Castro, 2008) y es tal que algunas investigaciones (Freudenthal, 1973; Polya 1979; Polya, 1954; Brown \& Walter, 1990; Ellerton 1986, Castro 2011; Espinoza, Lupiáñez \& Segovia, 2014) ponen de manifiesto su riqueza como foco de investigación.

En este sentido, ha sido abordada con el fin de mejorar las habilidades de resolución de problemas (Leung \& Silver, 1997), estudiar niños con talento matemático (Krutetskii, 1976; Ellerton, 1986; Kesan, Kaya \& Güvercin, 2010), tener una visión de la comprensión de los conceptos y procedimientos matemáticos de los estudiantes (English, 1997; Brown \& Walter 1993), estudiar cómo los estudiantes manejan y estructuran su propio conocimiento matemático (Pelczer \& Gamboa, 2008).

En relación con el talento matemático, econtramos que este tema es de interés para la comunidad de educadores e investigadores en Didáctica de la Matemática (Benavides, 2008), lo que puede evidenciarse, por ejemplo, en los grupos de estudio propuestos en el ICME 10 (TSG4) o el ICME 11 (TSG6). En este sentido, Castro (2008), menciona que los estudios sobre el talento matemático se han centrado en tres grandes focos de investigación: la caracterización del talento matemático, el establecer mecanismos de identificación y ofrecer alternativas de intervención. Centrándonos en la caracterización del talento matemático, algunos investigadores (Krutetskii, 1976; Greenes, 1981; Pasarín, Feijo, Díaz \& Rodríguez, 2004; Banfiel, 2005), han observado y analizado el pensamiento característico de estos estudiantes generalmente mediante tareas de resolución de problemas y concluyen que el razonamiento que muestran es muy diferente de aquellos estudiantes ordinarios en términos de velocidad y profundidad (Kesan, et al., 2010).

Otras investigaciones han caracterizado el talento matemático mediante tareas de invención de problemas. Al respecto se puede mencionar el estudio de Krutetskii (1976), quien 
reportó que los estudiantes con talento matemático podían ver aquellos problemas que surgen naturalmente de una información dada, mientras que los estudiantes con baja habilidad hacia la matemática no lo hicieron, incluso cuando el entrevistador les dio consejos.

En la investigación realizada por Ellerton (1986), se observó que los problemas inventados por los estudiantes con más habilidad requieren mayor dificultad de cálculo, presentan una mayor cantidad de operaciones, implican un sistema numérico más complejo y utilizan el lenguaje matemático con mayor fluidez que sus compañeros menos capaces. El estudio también concluye que hay poca evidencia que sugiera que los estudiantes menos hábiles planearan sus problemas, mientras que los producidos por estudiantes más hábiles presentaron mayor consistencia con el resto del problema, lo que sugiere cierta planificación. Además, se observó que los estudiantes más hábiles saben cómo resolver sus propios problemas, mientras que sus compañeros menos capaces no siempre saben por dónde empezar.

Silver \& Cai (1996), también propusieron este tipo de tareas a estudiantes con mayor y menor habilidad matemática. Los resultados indican que los estudiantes del grupo alto (mayor habilidad matemática) generaron no solo más problemas matemáticos, sino que también más problemas matemáticos complejos que sus compañeros (grupo bajo) y que el rendimiento de los estudiantes en la resolución de problemas tuvo una alta correlación con su rendimiento en el planteamiento del problemas. También se observó que los estudiantes del grupo alto plantearon una cantidad significativamente mayor de problemas matemáticos que implican dos o más relaciones semánticas que sus compañeros del grupo bajo.

Por último, Kesan et al. (2010), estudiaron el efecto de las actividades de invención de problemas en el desarrollo de habilidades matemáticas de 40 estudiantes con talento matemático. El estudio concluye que este tipo de actividades son efectivas para mejorar las habilides de los estudiantes en resolución de problemas, así como su rendimiento en matemática, especialmente para tareas no rutinarias y de composición abierta.

A pesar de estos antecedentes, existen pocos estudios que relacionen ambos tópicos, de manera que pongan de manifiesto las características particulares que presentan los estudiantes con talento matemático ante tareas de invención de problemas o si esta actividad puede emplearse como técnica diagnóstica. Por esta razón, nos centraremos en caracterizar la actuación de un grupo de estudiantes considerados con talento matemático, ante tareas semi- 
estructuradas de invención de problemas aritméticos, construidas especialmente para este estudio y compararlo con las actuaciones que presentan un grupo de estudiantes de un colegio público ante la misma tarea. Además nos interesa identificar indicios del uso de la invención de problemas como herramienta para identificar estudiantes con talento en matemática.

A continuación se tratan algunos conceptos relacionados con los sujetos con talento matemático, la invención de problemas matemáticos y los problemas aritméticos.

\section{Talento matemático}

Algunos autores sostienen que los estudiantes con talento presentan características que los diferencias del resto de sus compañeros. Por ejemplo, Greenes (1981) menciona que presentan un mayor ritmo de aprendizaje, excelente memoria y excepcionales capacidades verbales y de razonamiento y gran poder de abstracción. Pero, ¿quiénes son los estudiantes con talento?

$\mathrm{Al}$ respecto, se diferencian cinco nociones del talento orientadas en distintos aspectos: al logro o rendimiento, a lo innato, a la interacción entre lo innato y el medio ambiente, a modelos cognitivos y a modelos sistémicos (Villarraga, Martínez \& Benavides, 2004). En este estudio trataremos la noción de talento orientado al logro o rendimiento, ya que nos centramos en estudiar el rendimiento de estudiantes considerados con talento matemático.

De igual forma estudiaremos un talento específico, el talento matemático, por lo que adoptamos la definición de Passow (1993), para referirnos a los alumnos que han demostrado aptitudes específicas en el área de matemáticas. Esto porque uno de los grupos seleccionados está conformado por estudiantes que han demostrado, con base en pruebas de selección, aptitudes específicas en el área de matemáticas. En relación con las habilidades que caracterizan el desempeño de esos sujetos en matemáticas, Ramírez (2012) recoge los hallazgos de varios investigadores entre los que cita a Freiman (2006), quien propone habilidades como las siguientes: cuestionar con espontaneidad aspectos que van más allá de tareas escolares; transitar con facilidad entre diferentes estructuras matemáticas; focalizar los puntos clave de los problemas; desarrollar estrategias eficientes de resolución de problemas; producir ideas originales, valiosas y extensas, entre otras. Precisamente con esta última habilidad establecemos el vínculo con la invención de problemas. 
En cuanto a la identificación del talento matemático, se usan diversos métodos tanto de enfoque cualitativo como cuantitativos; sin embargo, los más utilizados han sido los test estandarizados, corriendo el peligro de rechazar a niños que deberían ser identificados como talentos matemáticos (Benavides, 2008). Por esto, investigaciones en España recomiendan emplear instrumentos adicionales a los que tradicionalmente son utilizados, con el objetivo de identificar un mayor número de estudiantes con talento (Hernández \& Gutiérrez, 2014).En este sentido, algunos autores (Krutetskii, 1976; Ellerton, 1986, Kesan et al., 2010) destacan el uso de la invención de problemas como una herramienta que podría emplearse tanto en la identificación de estudiantes con talento matemático, como en la comprensión de la naturaleza de las habilidades matemáticas de niños considerados con talento matemático. Por ejemplo, en el estudio de Getzels y Jackson (1962; citado en Silver, 1994) y Balka (1974), se utilizaron actividades de invención de problemas en el proceso para identificar individuos creativos, el cual es un rasgo presente en los sujetos con altas capacidades (Marugan, Carbonero, Torres \& León, 2012).

\section{Invención de problemas}

El término invención de problemas o planteamiento de problemas, también conocida en la literatura en inglés como "problem posing" (Kilpatrick, 1987; Brown \& Walter, 1993; Silver, 1994; English, 1997), consiste en la formulación de nuevos problemas, así como la reformulación de situaciones dadas (Silver, 1994; Silver \& Cai, 1996; English, 1997). En este sentido, Silver (1994) menciona que este proceso se puede llevar a cabo de tres formas diferentes: (a) antes de resolver un problema, cuando lo que se persigue no es la solución, sino la formulación de uno a partir de una situación o experiencia, (b) durante la solución de un problema complejo, reformulandolo en situaciones más sencillas, y (c) después de resolver un problema, cuando se busca modificar el objetivo, condición o pregunta del mismo, con el fin de generar nuevos problemas.

Por otra parte, se identifican tres formas en las cuales se podrían formular problemas: situación libre, situaciones semiestructuradas y situaciones estructuradas (Stoyanova, 1998). En la primera, los estudiantes no tienen restricciones para inventar problemas; mientras que en las situaciones semiestructuradas se les propone que planteen problemas con base en alguna experiencia o situación. Por último, las situaciones estructuradas, son aquellas en las que se reformulan los problemas dados o se cambia la condición del mismo. 
Así, consideramos que la invención de problema es un proceso matemático complejo, en el que se construyen uno o más problemas a partir de la interpretación personal o significado dado a una situación concreta o un problema previamente dado.

\section{Problemas aritméticos}

Dado que en este estudio se propusieron dos tareas de invención de problemas matemáticos aritméticos, consideramos pertinente exponer la noción de problema matemático que empleamos en nuestro estudio. Así, adoptamos la noción propuesta por Castro (1991), quien señala cinco componentes que debe incluir una situación para ser considerada un problema matemático: una proposición (enunciado oral o escrito), unos datos conocidos; una intención (movilizar una o más personas para que lo resuelvan), una meta (llegar a un resultado) y un proceso (modo de actuación para alcanzar el resultado).

Consideramos que un problema es aritmético si, siendo un problema matemático, proporciona información de carácter cuantitativo, la condición en el enunciado expresa relaciones cuantitativas entre los datos y la pregunta se refiere al cálculo de una o varias cantidades o relaciones entre cantidades (Puig \& Cerdán,1988). También consideraremos como aritmético, aquel problema que emplee incógnitas o ecuaciones siempre que la solución de este se base en una técnica aritmética o predominantemente aritmética (Gasco \& Villarroel, 2014).

En relación con su clasificación, Puig \& Cerdán (1988), mencionan que un problema aritmético se puede clasificar, según su estructura operatoria, en: aditivo de una etapa, multiplicativo de una etapa, aditivo de más de una etapa, multiplicativo de más de una etapa y problemas de varias operaciones combinadas. Estos últimos son también llamados problemas mixtos y son aquellos que combinan las estructuras aditivas y multiplicativas y su resolución requiere de más de una relación entre los datos (Castro, et al., 1997). Con respecto a las variables de estudio de los problemas aritméticos, Puig \& Cerdán (1988), destacan las variables sintácticas que están relacionadas con el orden y relaciones de las palabras y símbolos que contiene el enunciado. Por ejemplo: longitud del enunciado, complejidad gramatical, presentación de los datos, ubicación de la pregunta, etc. Castro (1995), menciona otro tipo de variable denominada proposición interrogativa, la cual se relaciona con la pregunta del problema y puede hacerse sobre una asignación o relación. Silver \& Cai (2005), agregan un tercer tipo de proposición interrogativa denominada condicional, en la cual la pregunta establece una condición entre dos elementos, por ejemplo: Si María recorrió 300 metro más que Pedro, ¿cuántos 
metros recorrió María? Estos autores asocian esta variable con la complejidad lingüística de un problema.

Por otra parte, Castro, Rico \& Gil (1992) destacan los datos numéricos en el enunciado, los cuales pueden distinguirse según el conjunto y tamaño de los números, la inclusión de datos superfluos, las operaciones necesarias para la obtención del resultado y el algoritmo empleado en cada operación. Castro et al., (1997), hace referencia a esta variable y menciona que si los cálculos necesarios para resolver un problema aritmético implican sólo adiciones y multiplicaciones entonces es un problema de dos procesos.

Por último, se debe considerar la componente semántica (Nesher, 1982; citado en Puig \& Cerdán, 1988), la cual se clasifica en cambio, combinación, comparación e igualación para los problemas aditivos y comparación multiplicativa, igualación, isomorfismo de medidas y producto de medidas para los problemas de estructura multiplicativa.

\section{Objetivos}

El objetivo general del estudio consiste en describir, analizar y caracterizar la capacidad de un grupo de estudiantes considerados con talento matemático en tareas de invención de problemas aritméticos verbales. Además, se pretende determinar indicios del uso de la invención de problemas como herramienta para identificar estudiantes con talento matemático.

Para ello se planteron los siguientes objetivos específico: (1) construir un instrumento de planteamiento de problemas con dos tareas o situaciones semiestructuradas de invención problemas aritméticos verbales; (2) definir un método analítico en base a categorías y variables que permitan caracterizar las producciones de ambos grupos de estudiantes ante la tarea de invención de problemas aritméticos, e (3) identificar diferencias entre los problemas inventados por ambos grupos con base en las categorías y componentes de análisis definidas.

\section{Método}

\section{Participantes}

Los sujetos de estudio corresponden a dos grupos de estudiantes españoles con características diferentes. El primero, denominado "grupo talento", está conformado por 21 estudiantes considerados con talento matemático que participaron en el proyecto ESTALMAT 
Andalucía $^{1}$ durante el curso 2010-2011 y que tienen edades comprendidas entre los 13 y los 15 años. Este proyecto pretende detectar y estimular durante dos años académicos el talento precoz en matemática de un grupo de alumnos de centros andaluces escogidos mediante la realización de pruebas de selección ${ }^{2}$. El segundo lo conforman 19 estudiantes de tercer grado del Instituto de Educación Secundaria Nazarí, ubicado en Salobreña, provincia de Granada, y que denominaremos "grupo estándar". Estos estudiantes tienen entre 14 y 15 años.

\section{Instrumento}

Se elaboró un cuestionario formado por dos tareas correspondientes a situaciones semiestructuradas de invención de problemas (Stoyanova, 1998), que permitieran a los estudiantes poner en práctica elementos que están relacionados con el talento, como son sus habilidades, conocimientos matemáticos y creatividad. Este último es considerado como uno de los rasgos que definen a la persona superdotada (Marugan, Carbonero, Torres \& León, 2012).

Para el diseño del instrumento se tomó en cuenta la clase de información que proporciona el problema, el tipo de información que permanece desconocida y que el contexto escolar presentado en la situación fuera familiar para los estudiantes (Moses, Bjork, \& Goldenberg, 1990). Además, se solicitó a los estudiantes plantear problemas que considerasen difíciles de resolver, ya que nos interesaba que tomaran la actividad como un reto y se esforzaran en poner en práctica sus conocimientos, habilidades, creatividad y experiencias previas cuando inventaran problemas. Esta indicación se ha empleado eficazmente en otras investigaciones, como la de Ayllón (2012). Este instrumento fue revisado y analizado por expertos en Didáctica de la Matemática y posteriormente se aplicó una prueba piloto con un grupo pequeño de estudiantes, quedando finalmente como sigue:

Tarea 1. De acuerdo con la información de la siguiente figura, inventa un problema matemático que te parezca difícil de resolver y que en su resolución se utilice una o varias de las operaciones de suma, resta, multiplicación o división. Si lo consideras necesario puedes agregar más datos o información.

\footnotetext{
${ }^{1}$ El programa ESTALMAT (Estímulo del Talento Matemático) es una iniciativa de la Real Academia de Ciencias Exactas, Físicas y Naturales de España, que se concreta en un programa formativo para escolares con talento matemático. Se pueden consultar sus bases en http://www.estalmat.org. La concreción del programa de la comunidad autónoma andaluza esta disponible en http://thales.cica.es/estalmat.

${ }^{2}$ http://thales.cica.es/estalmat/?q=node/39.

Electronic Journal of Research in Educational Psychology, 14(2), 368-392. ISSN:1696-2095. 2016. no. 39
}

http://dx.doi.org/10.14204/ejrep.39.15067 


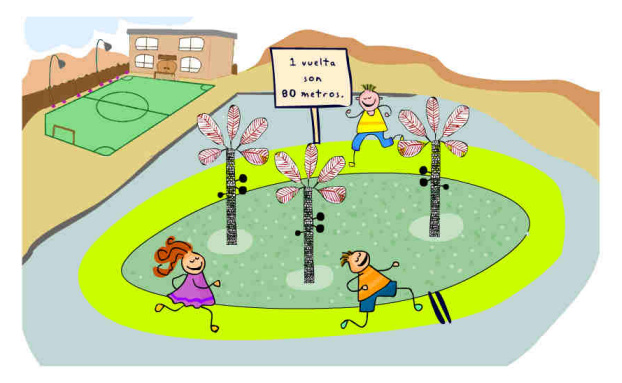

Figura 1. Imagen utilizada en la primera tarea ${ }^{3}$

Tarea 2. Con la siguiente información inventa un problema matemático que te parezca difícil de resolver y que en su resolución se utilice una o varias de las operaciones de suma, resta, multiplicación o división. Si lo consideras necesario puedes agregar más datos o información.

"Un tren con cuatro vagones para pasajeros sale de una estación a las 9:00 h con destino a Málaga. El tren tiene una capacidad máxima para 294 pasajeros”.

\section{Descripción de las categorías y componentes de análisis}

Para elaborar las categorías de análisis consideramos las características de esta investigación y realizamos una revisión de las variables de estudio de los problemas aritméticos propuestas por Puig \& Cerdán (1988); Castro (1995); Castro et al., (1992) y los esquemas empleados por Leung \& Silver (1997); Silver \& Cai (2005; 1996); Cázares (2000); Ayllón (2012). Así, definimos tres categorías de análisis y en cada una de ellas variables de estudio que se explican con mayor detalle en Espinoza, Lupiáñez \& Segovia (2015).

En la primera categoría, denominada estructura sintáctica, se estudió la longitud del enunciado, tipo de proposición interrogativa y tipo de números empleados. La longitud del enunciado fue analizada de acuerdo a la cantidad de proposiciones presentes, las cuales corresponden a expresiones explícitas en el texto que asignan un valor numérico o una cantidad a una variable, o bien, establece una relación cuantitativa entre dos variables. El tipo de proposición interrogativa se relaciona con la pregunta del problema y se clasifican en proposiciones de asignación, condicionales o relacionales (Silver \& Cai, 2005). Los números pueden ser

\footnotetext{
${ }^{3}$ Figura obtenida de http://ntic.educacion.es/w3/eos/MaterialesEducativos/mem2009/problematic/menuppal.html. 


\section{Johan Espinoza et al.}

enteros o racionales, estos últimos en sus distintos sistemas de representación, expresión decimal o fraccionaria.

La segunda categoría, llamada estructura matemática, fue analizada de acuerdo con el tipo de estructura operatoria (aditiva, multiplicativa o mixta), y número de etapas o pasos en cada estructura para resolver el problema, tipos de operación aritmética (suma, resta, multiplicación o división), cantidad de procesos de cálculo distintos implicados en la solución del problema, según Castro, et al (1997) y cantidad de pasos distintos para resolver el problema.

Por último, en la categoría de estructura semántica, los problemas fueron estudiados en relación a su estructura semántica (Cambio, Combinación, igualación y Comparación para los problemas aditivos e Isomorfismo de medidas, Comparación y Producto de medidas para los multiplicativos) y cantidad de relaciones semánticas distintas presentes en el enunciado.

Las componentes de las tres categorías y la solución del problema permiten caracterizar los enunciados de los estudiantes y evaluar su riqueza. Para estudiar si existen diferencias significativas entre las producciones del grupo talento y el grupo estándar, hemos aplicado el estadístico Chi-cuadrado por la corrección por continuidad de Yates, ya que se cumple la exigencia de que los valores esperados de al menos el $80 \%$ de las celdas de contingencia, sean mayores que 5. Para efectuar ese análisis hemos empleado un software comercial (SPSS para Windows, versión 19; SPSS Inc., Chicago, IL, Estados Unidos).

\section{Esquema para valorar la solución de las producciones de los estudiantes}

Todas las producciones de los estudiantes fueron clasificadas en resolubles y no resolubles. Dentro de éstos encontramos no resolubles que presentaron características importantes de analizar. Por ello los clasificamos como incompletos (Puig \& Cerdán, 1988) y los distinguimos de aquellos que presentan incompatibilidad matemática de tipo numérico o conceptual. Los problemas matemáticos resolubles y no resolubles incompletos o que presentan incompatibilidad matemática de tipo numérica se analizaron desde su estructura sintáctica, semántica y matemática explicado anteriormente. Mientras que los problemas matemáticos que presentan incompatibilidad matemática de tipo conceptual fueron analizados sólo desde su estructura sintáctica, pues no era posible analizar las otras dos estructuras. La figura 2 muestra el esquema utilizado para valorar las producciones de los estudiantes. 


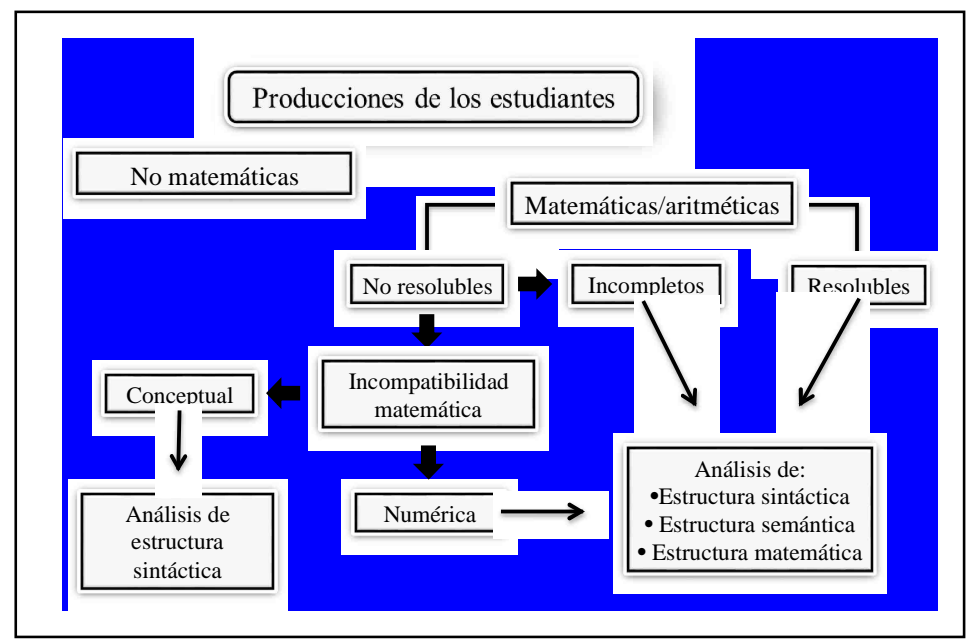

Figura 2. Esquema para valorar las producciones de los estudiantes

\section{Procedimiento}

Una vez fijado los objetivos y confeccionado el instrumentos y el esquema para valorar las producciones de los estudiantes, se solicitó la colaboración de los profesores de ambos grupos para aplicar en un tiempo de 20 minutos y por separado el instrumento de invención de problemas. Éste se aplicó de igual forma en los dos grupos y consistió en que el entrevistador-investigador entregó las dos hojas con las tareas de invención de problemas a cada estudiante y les indicó que la actividad propuesta formaba parte de una investigación que se realiza en el Departamento de Didáctica de la Matemática de la Universidad de Granada, con relación a los procesos de invención de problemas matemáticos.

\section{Resultados}

En este apartado se presentan los principales resultados obtenidos de las producciones de ambos grupos de estudiantes a las dos tareas planteadas. A continuación se muestran las características generales de los problemas inventados y seguidamente se exponen los resultados obtenidos según la estructura sintáctica, matemática y semántica de los problemas.

\section{Características generales de los problemas inventados}

En primera instancia resultó que todos los enunciados inventados por los estudiantes son problemas matemáticos, de los cuales el $65 \%$ son resolubles. Es interesante hacer notar que los estudiantes del grupo estándar plantearon una mayor proporción de problemas resolubles $(74 \%)$ que el grupo talento (57\%). Este resultado sorprende ya que se espera que ocurra lo contrario; a falta de la realización de otro estudio más amplio que los confirme, pueden ser 
varios los factores que inciden: mejor actitud del grupo talento ante las matemáticas, menor ansiedad y miedo a equivocarse, el no tener que resolver los problemas que inventaban, etc.

También se obtuvo que los problemas no resolubles por incompatibilidad matemática representan el 22,5\% (18 problemas, de los cuales 12 fueron planteados por el grupo talento y 6 por el grupo estándar) y los problemas incompletos 12,5\% (10 problemas, de los cuales 6 fueron inventados por el grupo talento y 4 por el grupo estándar). Estos dos tipos de problemas representan el 35\% de los problemas matemáticos producidos por los estudiantes.

Tabla 1. Distribución de los problemas de acuerdo con su resolubilidad y grupo al que pertenece el estudiante

\begin{tabular}{cccccccc}
\hline & \multirow{2}{*}{$\begin{array}{c}\text { Problemas resolu- } \\
\text { bles }\end{array}$} & & \multicolumn{3}{c}{ Problemas no resolubles } & \\
\cline { 2 - 6 } & Frecuencia & $\%$ & Frecuencia & $\%$ & Frecuencia & $\%$ & \\
\cline { 2 - 6 } & 24 & 57,1 & 6 & 14,3 & 12 & 28,6 & 42 \\
Grupo talento & 28 & 73,7 & 4 & 10,5 & 6 & 15,8 & 38 \\
Grupo estándar & 52 & 65,0 & 10 & 12,5 & 18 & 22,5 & 80 \\
Total & 52 & & & & & & \\
\hline
\end{tabular}

Un problema planteado que presentó incompatibilidad matemática de tipo numérica porque 229 no es divisible por 3 es el siguiente: De la estación de tren de Madrid sale un tren con cuatro vagones a las 9:00 h con destino a Málaga. Todos los pasajes están vendidos (294) pero en un último momento uno de los vagones que tiene capacidad para 65 pasajeros sufre una serie de desperfectos por lo que debe quedarse en la estación. Si los restantes vagones tienen la misma capacidad. ¿Cuánto pasajeros pueden subir a cada vagón?

Un ejemplo de problema clasificado como incompleto porque no indica el total de personas que quedaron en el tren luego de la última parada es el siguiente: En este viaje va lleno. En una primera parada se bajan 2 parejas, una con un hijo más que la otra, y suben un número de personas tal que quedan 290. En la segunda parada bajan 10 parejas y suben 15 personas, y en la última antes de llegar, bajan 3 personas y suben el triple de niños que tenían las dos primeras parejas juntas. ¿Cuántas personas subieron en la primera parada y cuántos niños tenían cada pareja (de la $1^{\circ}$ parada)?

Dado que ambos grupos plantearon problemas no resolubles que presentan características interesantes de analizar, consideramos convenientes estudiar este tipo de problemas con 
base en cada una de las variables de estudio empleadas en la investigación.

\section{Análisis según la estructura sintáctica}

Longitud del enunciado. Resultó que la cantidad promedio de proposiciones presentes en los problemas es mayor en el grupo talento $(5,27)$ que en el grupo estándar $(3,44)$. La siguiente tabla muestra la caracterización de los problemas de acuerdo con esta variable

Tabla 2. Distribución de los problemas de acuerdo con la cantidad de proposiciones, tarea y grupo al que pertenece el estudiante

\begin{tabular}{lcccc|cccc}
\hline \multirow{2}{*}{ Cantidad de proposiciones } & \multicolumn{4}{c|}{ Grupo talento } & \multicolumn{4}{c}{ Grupo estándar } \\
\cline { 2 - 9 } & T1 & T2 & Total & $\%$ & T1 & T2 & Total & $\%$ \\
\hline Una o dos proposición & 5 & 0 & 5 & 11,9 & 6 & 2 & 8 & 21,1 \\
Tres proposiciones & 2 & 1 & 3 & 7,1 & 5 & 5 & 10 & 26,3 \\
Cuatro proposiciones & 3 & 2 & 5 & 11,9 & 6 & 2 & 8 & 21,1 \\
Cinco o seis proposiciones & 7 & 5 & 12 & 28,6 & 2 & 7 & 9 & 23,7 \\
Siete o más proposiciones & 4 & 13 & 17 & 40,5 & 0 & 3 & 3 & 7,9 \\
\multicolumn{1}{r}{ Total } & 21 & 21 & 42 & 100,0 & 19 & 19 & 38 & 100,0 \\
\hline
\end{tabular}

La tabla 2 muestra que el $69,1 \%$ de los problemas inventados por el grupo talento están formados por cinco o más proposiciones, mientras que el grupo estándar planteó el 31,6\% con dicha característica. También se observa que aproximadamente la mitad de los problemas inventados por el grupo estándar $(47,4 \%)$, poseen tres o menos proposiciones, en contraste con el grupo talento quienes plantearon el $19 \%$ con esta característica.

Por otra parte, resultó que el promedio de la cantidad de proposiciones presentes en los problemas no resolubles $(5,58)$ es mayor que en los resolubles $(5,04)$. Esto se evidenció en el planteamiento de una mayor proporción de problemas no resolubles que presentan cinco o más proposiciones $(77,8 \%)$, que resolubles con la misma característica $(62,5 \%)$.

El análisis Chi-Cuadrado ha mostrado que las diferencias entre ambos grupos son significativas, pues el p-valor asociado es 0.004 y por tanto se debe rechazar la hipótesis nula. Es decir, las variables Grupo y Número de proposiciones son dependientes.

Tipo de proposición interrogativa. Con respecto a esta variable, resultó que la mayoría de las proposiciones interrogativas que plantearon los estudiantes del grupo talento y estándar son de asignación (52,4\% y 60,5\% respectivamente). El siguiente es un ejemplo de problema que presenta una proposición interrogativa de asignación relacionado con la segunda tarea: $A$ 
las 9:00 de la mañana sale un tren con 50 pasajeros, a las once vuelve con 70 pasajeros, vuelve a salir y vuelve con 30 pasajeros. ¿Cuántos pasajeros han entrado y salido en total?

Tipos de números empleados. Se observó que ambos grupos prefirieron emplear números naturales en el planteamiento de su problema (97,6\% en el grupo talento y $97,4 \%$ grupo estándar). También resultó que el 43,9\% de los problemas planteados por el grupo talento presentan el uso de número racionales expresados tanto en notación decimal como fraccionaria; mientras que el grupo estándar planteó 18,5\% de problemas con esta carácterística. Además, el grupo talento planteó casi el doble de proporción de problemas con dos o más tipos de números que el grupo estándar, los cuales corresponden a 34,1\% y 18,4\% respectivamente.

\section{Análisis según la estructura matemática}

El análisis en esta categoría fue aplicado a 78 problemas, ya que dos que presentaron incompatibilidad matemática son imposibles de resolver incluso con información adicional.

Tipo de estructura operatoria y cantidad de etapas para resolver el problema. Resultó que la mayoría de problemas planteados por ambos grupos son de estructura mixta; sin embargo, el grupo talento planteó una mayor proporción (80\%) que sus compañeros del grupo estándar (55,3\%). En contraste, el grupo estándar planteó una proporción mayor de problemas de estructura multiplicativa $(31,5 \%)$ y aditiva $(13,1 \%)$, que sus compañeros del grupo talento (17,5\% y 2,5\% respectivamente). Además, se encontró que el 97,5\% y 94,8\% de los problemas planteados por el grupo talento y estándar, respectivamente, son de más de una etapa. La siguiente tabla muestra los resultados obtenidos de acuerdo a esta variable.

Tabla 3. Distribución de los problemas en relación con la estructura operatoria, número de etapas, tarea y grupo al que pertenece el estu-

\begin{tabular}{|c|c|c|c|c|c|c|}
\hline \multirow{2}{*}{$\begin{array}{l}\text { Estructura operatoria } \\
\text { y número de etapas }\end{array}$} & \multicolumn{3}{|c|}{ Grupo talento } & \multicolumn{3}{|c|}{ Grupo estándar } \\
\hline & $\mathrm{T} 1$ & $\mathrm{~T} 2$ & $\%$ & $\mathrm{~T} 1$ & $\mathrm{~T} 2$ & $\%$ \\
\hline Estructura mixta & 12 & 20 & 80,0 & 8 & 13 & 55,3 \\
\hline Multiplicativa de dos o más etapas & 6 & 0 & 15,0 & 9 & 2 & 28,9 \\
\hline Aditiva de dos o más etapas & 0 & 1 & 2,5 & 1 & 3 & 10,5 \\
\hline Multiplicativa de una etapa & 1 & 0 & 2,5 & 1 & 0 & 2,6 \\
\hline Aditiva de una etapa & 0 & 0 & 0,0 & 0 & 1 & 2,6 \\
\hline Total & 19 & 21 & 100,0 & 19 & 19 & 100,0 \\
\hline
\end{tabular}


En este caso, agrupando los valores de la variable estructura en aditiva, multiplicativa y mixta el p-valor asociado para la Chi-cuadrado es 0.045 , lo que pone de manifiesto también diferencias significativas entre ambos grupos. En relación con la resolubilidad del problema y esta variable, resultó que ambos grupos plantearon más problemas no resolubles con estructura mixta que problemas resolubles con dicha característica. Sin embargo, esto no fue así en los problemas de estructura multiplicativa y aditiva, ya que la mayor proporción de estos son problemas resolubles.

Tipo de operación y cantidad de procesos implicados en la solución del problema. La siguiente tabla muestra la clasificación de los problemas de acuerdo con el tipo de operación.

Tabla 4. Distribución de los problemas de acuerdo con el tipo de operación y grupo al que pertenece el estudiante

\begin{tabular}{|c|c|c|c|c|}
\hline Tipo de operación & $\begin{array}{l}\text { Grupo } \\
\text { talento }\end{array}$ & $\%$ & $\begin{array}{l}\text { Grupo } \\
\text { estándar }\end{array}$ & $\%$ \\
\hline Suma & 1 & 2,5 & 3 & 7,9 \\
\hline Multiplicación & 2 & 5,0 & 4 & 10,5 \\
\hline Suma-Multiplicación-División & 4 & 10,0 & 1 & 2,6 \\
\hline Suma-Resta-Multiplicación & 5 & 12,5 & 3 & 7,9 \\
\hline Multiplicación-División & 6 & 15,0 & 7 & 18,4 \\
\hline Suma-Multiplicación & 9 & 22,5 & 10 & 26,3 \\
\hline Suma-Resta-Multiplicación-División & 9 & 22,5 & 3 & 7,9 \\
\hline Otra & 4 & 10,0 & 7 & 18,4 \\
\hline Total & 40 & 100,0 & 38 & 100,0 \\
\hline
\end{tabular}

De acuerdo con la tabla 4, el $60 \%$ de los problemas del grupo talento implicaban el uso de multiplicación-división, suma-multiplicación y suma-resta-multiplicación-división. En el grupo estándar, el 55,2\% de los problemas requieren las operaciones de multiplicación, multiplicación-división y suma-multiplicación. En este caso el p-valor que arroja la Chicuadrado es de 0.031 lo que muestra la significatividad de las diferencias entre ambos grupos.

Con respecto a la cantidad de procesos (Castro et al. 1997) implicados en la solución del problema (tabla 5), resultó que el grupo talento y estándar plantearon respectivamente el $92,5 \%$ y $81,6 \%$ de los problemas con dos o más procesos. También se observó que el 47,5\% de los problemas del grupo talento presentan tres o más procesos distintos; mientras que el 21,1\% de los problemas del grupo estándar presentan tal cantidad de procesos. Cabe resaltar que los estudiantes del grupo talento y estándar plantearon una proporción similar de problemas con dos procesos o tres procesos ( $70 \%$ y $73,7 \%$ respectivamente). En el test Chi- 
cuadrado el p-valor resultante es de 0.018 lo que indica diferencias significativas entre ambos grupos. Por último, consideramos que no existen diferencias en la cantidad de procesos implicados en los problemas resolubles y no resolubles.

Tabla 5. Clasificación de los problemas de acuerdo con la cantidad de procesos distintos implicados en la solución, tarea y grupo al que pertenece el estudiante

\begin{tabular}{|c|c|c|c|c|c|c|}
\hline \multirow{2}{*}{$\begin{array}{c}\text { Cantidad de procesos } \\
\text { distintos }\end{array}$} & \multicolumn{3}{|c|}{ Grupo talento } & \multicolumn{3}{|c|}{ Grupo estándar } \\
\hline & $\mathrm{T} 1$ & $\mathrm{~T} 2$ & $\%$ & $\mathrm{~T} 1$ & $\mathrm{~T} 2$ & $\%$ \\
\hline Un proceso & 2 & 1 & 7,5 & 4 & 3 & 18,4 \\
\hline Dos procesos & 11 & 7 & 45,0 & 11 & 12 & 60,5 \\
\hline Tres procesos & 4 & 6 & 25,0 & 3 & 2 & 13,2 \\
\hline Cuatro procesos & 2 & 7 & 22,5 & 1 & 2 & 7,9 \\
\hline Total & 19 & 21 & 100,0 & 19 & 19 & 100,0 \\
\hline
\end{tabular}

Cantidad de pasos distintos para resolver el problema. Observamos que el promedio de pasos para resolver el problema es mayor en el grupo talento $(3,95)$ que en el grupo estándar $(2,92)$. La tabla 6 muestra la distribución de los problemas de acuerdo con esta variable.

Tabla 6. Clasificación de los problemas de acuerdo con la cantidad de pasos distintos para resolverlo, tarea y grupo al que pertenece el estudiante

\begin{tabular}{lccc|ccc}
\hline Cantidad de pasos & \multicolumn{3}{c|}{ Grupo talento } & \multicolumn{3}{c}{ Grupo estándar } \\
\cline { 2 - 7 } \multicolumn{1}{c}{ Distintos } & T1 & T2 & $\%$ & T1 & T2 & $\%$ \\
\hline Un paso & 1 & 1 & 5,0 & 2 & 1 & 7,9 \\
Dos pasos & 4 & 2 & 15,0 & 8 & 5 & 34,2 \\
Tres pasos & 3 & 2 & 12,5 & 4 & 6 & 26,3 \\
Cuatro pasos & 2 & 4 & 15,0 & 3 & 5 & 21,1 \\
Cinco o más pasos & 9 & 12 & 52,5 & 2 & 2 & 10,5 \\
$r$ Total & 19 & 21 & 100,0 & 19 & 19 & 100,0 \\
\hline
\end{tabular}

De acuerdo con la tabla, la diferencia en los promedios también se reflejó en la cantidad de problemas que requieren cuatro o más pasos para ser resueltos, puesto que el grupo talento planteó el 67,5\% de este tipo y el grupo estándar 31;6\%. Otro aspecto interesante es que los estudiantes del grupo estándar plantearon una gran cantidad de problemas que requieren entre dos y cuatro pasos para ser resueltos $(81,6 \%)$; en contraste con el grupo talento donde el $42,5 \%$ presentan dicha característica. Estadísticamente hay diferencias significativas entre ambos grupos con un p-valor para el test Chi-cuadrado de 0.003 . 
En relación con la resolubilidad del problema, se encontró que en el caso del grupo talento el promedio de pasos requeridos para resolver los problemas resolubles $(4,04)$ es mayor que en los no resolubles $(3,81)$, en contraste con el grupo estándar donde el promedio de pasos es mayor en los no resolubles $(3,4)$ que en los resolubles $(2,75)$. Además, el grupo estándar planteó una mayor proporción de problemas no resolubles con más de cuatro pasos (50\%), que problemas resolubles con la misma característica (26\%).

\section{Análisis según la estructura semántica}

En esta categoría, al igual que en la anterior, sólo se analizaron 78 problemas.

Estructura semántica de los problemas aditivos. Al estudiar solamente los problemas de estructura aditiva, resultó que el grupo talento planteó sólo un enunciado con esta característica, el cual presenta la estructura semántica de cambio; mientras que en el grupo estándar encontramos 8 problemas de este tipo, de los cuales 4 presentan la estructura semántica de combinación, 3 de cambio y uno de comparación. Por otra parte, de los 59 problemas de estructura aditiva o mixta (33 inventados por el grupo talento y 26 por el estándar), se encontró que ambos grupos prefirieron plantear problemas de combinación, seguido de problemas que incluyen la componente semántica de cambio.

Estructura semántica de los problemas multiplicativos. Observamos que las estructuras semánticas más utilizadas por el grupo talento fueron la de producto de medidas $(71,4 \%)$ e isomorfismo de medida (57,1\%). En el caso del grupo estándar, el 91,7\% de los problemas multiplicativos presentan la relación semántica de isomorfismo de medida y el $25 \%$ producto de medidas. Por otra parte, de los 72 problemas de estructura multiplicativa o mixta (39 planteados por el grupo talento y 33 por el grupo estándar), se encontró que ambos grupos prefirieron plantear problemas que incluyeran la componente semántica de isomorfismo de medida, seguida de producto de medidas y en menor proporción comparación multiplicativa.

Relaciones semánticas implicadas en los problemas mixtos. Encontramos que el $66 \%$ de los problemas mixtos planteados por el grupo estándar incluyen las componentes semánticas combinación-isomorfismo de medidas o cambio-isomorfismo de medidas,; mientras que el grupo talento planteó sólo el 13\% con estas características. En el caso del grupo talento no es posible establecer una mayoría del uso específico de combinaciones de estructuras semánticas aditivas y multiplicativas implicadas en los problemas mixtos; sin embargo, la combina- 
ción que tiene mayor frecuencia (30\%) es cambio-combinación-isomorfismo de medidas o cambio-comparación aditiva-isomorfismo de medidas.

Cantidad de relaciones semánticas distintas. Observamos que el promedio de la cantidad de relaciones semánticas distintas es mayor en grupo talento $(2,83)$ que en el grupo estándar $(1,89)$. En la siguiente tabla se profundiza un poco más con respecto a esta variable.

Tabla 7. Clasificación de los problemas de acuerdo con la cantidad de relaciones de estructura semántica diferentes, tarea y grupo al que pertenece

\begin{tabular}{lcccc|cccc}
\hline \multicolumn{1}{c}{ el estudiante } \\
Cantidad de relaciones & \multicolumn{3}{c}{ Grupo talento } & \multicolumn{4}{c}{ Grupo estándar } \\
\cline { 2 - 10 } \multicolumn{1}{c}{ semánticas distintas } & T1 & T2 & Total & $\%$ & T1 & T2 & Total & $\%$ \\
\hline Una relación & 3 & 1 & 4 & 10,0 & 7 & 3 & 10 & 26,3 \\
Dos relaciones & 6 & 4 & 10 & 25,0 & 10 & 12 & 22 & 57,9 \\
Tres relaciones & 9 & 8 & 17 & 42,5 & 2 & 4 & 6 & 15,8 \\
Cuatro relaciones & 1 & 6 & 7 & 17,5 & 0 & 0 & 0 & 0,0 \\
Cinco o más relaciones & 0 & 2 & 2 & 5,0 & 0 & 0 & 0 & 0,0 \\
$\quad$ Total & 19 & 21 & 40 & 100,0 & 19 & 19 & 38 & 100,0 \\
\hline
\end{tabular}

La tabla 7 muestra que el grupo talento planteó una proporción mayor (65\%) de problemas con tres o más relaciones semánticas distintas que sus compañeros del grupo estándar $(15,8)$. Además, todos los problemas del grupo estándar poseen tres o menos relaciones semánticas y un porcentaje significativo presentan dos o menos relaciones semánticas distintas $(84,2 \%)$. También resultó que el grupo talento y estándar plantearon un porcentaje similar de problemas que presentan dos o tres relaciones semánticas distintas $(67,5 \%$ y 73,7 respectivamente). El p-valor asociado a la prueba Chi-cuadrado es de 0.00 lo que muestra la significatividad de las diferencias entre ambos grupos.

\section{Discusión y conclusiones}

En primera instancia, consideramos que el instrumento de invención de problemas y el esquema analítico empleado en esta investigación permitieron describir y explorar la actuación de un grupo de estudiantes considerados con talento matemático. Además, las tres categorías de análisis empleadas y la solución de cada problema dan lugar a diez componentes cuyos valores caracterizan la riqueza de los problemas inventados por los sujetos: longitud del enunciado, proposición interrogativa, números empleados, estructura operatoria, número de etapas, tipo de operación, cantidad de procesos, cantidad de pasos en la resolución, estructura 
semántica y número de relaciones semánticas. Además, se han constatado diferencias estadísticamente significativas entre las producciones de ambos grupos en estudio.

En este sentido, concluimos que los problemas inventados por el grupo talento presentan mayor riqueza que los planteados por el grupo estándar, ya que están conformados por una mayor cantidad de proposiciones y tipos de números, requieren más pasos y procesos de cálculo distintos para ser resueltos y presenta una mayor cantidad de relaciones semánticas distintas. Este resultado es similar al obtenido por Ellerton (1986), pues los problemas inventados por los estudiantes más hábiles requirieron mayor dificultad de cálculo, presentaron una mayor cantidad de operaciones e implicaron un sistema numérico más complejo que los inventados por sus compañeros con menor habilidad.

Con respecto a la resolubilidad de los problemas, concluimos que los no resolubles inventados por el grupo talento presentan una mayor cantidad de proposiciones, tipos de números y relaciones semánticas distintas que los resolubles; sin embargo, estos últimos requieren una mayor cantidad de pasos para ser resueltos. También se observó que este grupo planteó una mayor proporción de problemas no resolubles con estructura mixta y que presentan proposiciones interrogativas de asignación.

Consideramos que esto pudo suceder porque los estudiantes intentaron agregar condiciones al problema con la intención de hacerlo más difícil, sin verificar que fuera resoluble. A pesar de esto, creemos que es importante analizarlos, ya que inventar un problema resoluble es una parte más de la complejidad que conlleva la actividad de inventar un problema difícil. Además, una gran proporción de estos presentaron incompatibilidad matemática de tipo numérica, pero son enunciados de una gran riqueza según las variables de estudio consideradas.

Por otra parte, tras analizar las producciones de los estudiantes del grupo talento con base en el esquema analítico y las tres categorías de análisis definidas en esta investigación, y tomando en cuenta las condiciones y los resultados de este estudio, concluimos que un estudiante con talento en matemáticas se puede caracterizar por:

a) Inventar una gran cantidad de problemas no resolubles.

b) Incluir en el enunciado del problema cinco o más proposiciones.

c) Emplear números naturales y en menor proporción números racionales. 
d) Emplear dos tipos de números distintos, ya sean naturales o racionales expresados en notación decimal y/o fraccionaria.

e) Incluir como pregunta del problema proposiciones interrogativas de asignación.

f) Combinar la estructura aditiva y multiplicativa para plantear problemas de estructura mixta.

g) Incluir las relaciones semánticas de combinación y producto de medidas.

h) Plantear tres o más relaciones semánticas distintas.

i) Inventar problemas que requieren cuatro o más pasos para resolverlo.

j) Plantear problemas que presentan dos o más procesos de cálculo distintos en su solución y en menor proporción tres o más procesos.

Por último, concluimos que las actividades de invención de problemas pueden ser consideradas en la identificación de estudiantes con talento en matemáticas. Esta afirmación se sostiene ya que los estudiantes del grupo talento mostraron una mayor capacidad de invención de problemas en cuanto a la riqueza de los mismos que sus compañeros del grupo estándar. Además, percibimos una mayor dificultad al resolver los problemas planteados por este grupo, puesto que al leer el enunciado no se identificaba de forma inmediata una solución al mismo.

\section{Referencias}

Ayllón, M. (2012). Invención-Resolución de problemas por alumnos de educación primaria. Tesis Doctoral. Granada: Universidad de Granada.

Banfield, T. (2005). Ability grouping for mathematically gifted adolescent boys. International Journal, 6(2), 141-149.

Benavides, M. (2008). Caracterización de sujetos con talento en resolución de problemas de estructura multiplicativa. Tesis doctoral. Granada: Universidad de Granada.

Brown, S. \& Walter, M. (1990). The Art of problem posing. New Jersey: Lawrence Erlbaum Associates.

Brown, S. \& Walter, M. (1993). Problem posing. New Jersey: Lawrence Erlbaum Associates.

Castro, E. (1995). Niveles de comprensión en problemas verbales de comparación multiplicativa. Tesis doctoral. Granada: Comares.

Castro, E. (1991). Resolución de problemas aritméticos de comparación multiplicativa. Memoria de Tercer Ciclo. Granada: Universidad de Granada. 
Castro, E., Rico, L. \& Gil, F., (1992). Enfoque de investigación en problemas verbales aritméticos aditivos. Enseñanza de las ciencias, 10(3), 243-253.

Castro, E., Castro, E., Rico, L., Gutiérrez, J., Tortosa, A., Segovia, I., et al. (1997). Problemas aritméticos compuestos de dos relaciones. En L. Rico y M. Sierra (Eds.), Primer simposio nacional de la SEIEM (pp. 63-76). Granada: SEIEM.

Castro, E. (2008). Resolución de problemas: ideas, tendencias e influencias en España. En R. Luengo, B. Gómez, M. Camacho \& L. Blanco (Eds.), Investigación en Educación Matemática XII (pp. 113-140). Badajoz: Sociedad Española de Investigación en Educación Matemática, SEIEM.

Castro, E. (2011). La invención de problemas y sus ámbitos de investigación. En J.L Lupiáñez, M.C Cañadas, M. Molina, M. Palarea y A. Maz (Eds.), Investigaciones en Pensamiento Numérico y Algebraico e Historia de la Matemática y Educación Matemática (pp 1-15). Granada, Departamento de Didáctica de la Matemática, Universidad de Granada.

Cázares, J. (2000). La invención de problemas en escolares de primaria: un estudio evolutivo. Memoria de tercer ciclo. Granda: Universidad de Granada.

Ellerton, N. (1986). Children's made up mathematics problems. A new perspective on talented mathematicians. Educational Studies in Mathematics, 17(3), 261-271. doi: 10.1007/BF00305073

English, L. (1997). The development of fifth-grade children's problem-posing abilities. Educational Studies in Mathematics, 34(3), 183-217. doi:10.1023/A:1002963618035

Espinoza, J., Lupiáñez J. L. \& Segovia, I. (2014). La invención de problemas y sus ámbitos de investigación en Educación Matemática. Revista digital Matemática, Educación e Internet (14)2, 1-12. doi:10.18845/rdmei.v14i2.1664

Espinoza, J., Lupiáñez J. L. \& Segovia, I. (2015). Un esquema para analizar los enunciados de los estudiantes en contextos de invención de problemas. Revista Unciencia (29)1, 5881. doi:10.15359/ru.29-1.4

Freiman, V. (2006). Problems to discover and to boost mathematical talent in early grades: A Challenging Situations Approach. The Montana Mathematics Enthusiast, 3(1), 51-75.

Freudenthal, H. (1973). Mathematics as an educational task. Dordrecht: Reidel.

Gasco, J. \& Villarroel, J. D. (2014). La motivación en la resolución de problemas aritméticoalgebraicos. Un estudio con alumnado de educación secundaria. Electronic Journal of research in Educational Psychology, 12(1), 83-106. doi:10.14204/ejrep.32.13076 
Greenes, C. (1981). Identifying the gifted student in mathematic. Arithmetic Teacher, 28(8), 14-17.

Hernández, D. \& Gutiérrez, M. (2014). El estudio de la alta capacidad intelectual en España: Análisis de la situación actual. Revista de Educación, 364, 251-272. doi:10.4438/1988-592X-RE-2014-364-261

Kilpatrick, J. (1987). Problem formulating: Where do good problems come from? En A. Shoenfeld (Ed.) Cognitive science and mathematics education. (pp. 123-148). New Jersey: Lawrance Erlbaum Associates.

Krutetskii, V. A. (1976). The psychology of mathematical abilities in school children. Chicago: University of Chicago Press.

Kesan, C., Kaya, D. \& Güvercin, S. (2010). The Effect of Problem Posing Approach to the Gifted Student's Mathematical Abilities. International Online Journal of Educational Sciences, 2(3), 677-687.

Leung, S. \& Silver, E. (1997). The role of task format, mathematics knowledge, and creative thinking on the arithmetic problem posing of prospective elementary school teachers. Mathematics Education Research Journal, 9(1), 5-24. doi:10.1007/BF03217299

Marugan, M., Carbonero M. A., Torres, M. \& Leon, B. (2012). Análisis de las relaciones entre creatividad y altas capacidades en primaria y secundaria. Electronic Journal of Research in Educational Psychology, 10(3), 1081-1098.

Moses, B., Bjork, E. \& Goldenberg, E. R. (1990). Beyond problem solving: problem posing. En T. J. Cooney y C. R. Hirsch (eds.), Teaching and Learning Mathematics in the 1990s (pp. 83-91). Reston, VA: National Council of Teachers of Mathematics.

National Council of Teachers of Mathematics. (1980). An Agenda for Action: Recommendations for School Mathematics of the 1980s. Reston, VA: Autor.

National Council of Teachers of Mathematics. (2000). Principles and Standards for School Mathematics. Reston, VA: Autor.

Pasarín, M. J., Feijoo, M., Díaz, O. \& Rodríguez, L. (2004). Evaluación del talento matemático en educación secundaria. Faísca, Revista de altas capacidades, 11, 83-102.

Passow, A. (1993). National/State Policies Regarding Education of the Gifted. En K.Heller, F. Monks y A. Passow (Eds.), International Handbook of Research and Development of Giftedness and Talent (pp. 29-46). Oxford: Pergamon Press.

Pelczer, I. \& Gamboa F. (2008). Problem posing strategies of mathematically gifted students. En R. Leikin (Ed.), Proceedings of the 5th International Conference on Creativity in 
Mathematics and the Education of Gifted Students (pp 193-199). Tel Aviv: Center for Educational Technology.

Polya, G. (1954). Mathematics and plausible reasoning. Princeton, NJ: Princeton University Press.

Polya, G. (1979). Cómo plantear y resolver problemas. México: Trillas.

Puig, L. \& Cerdán, F. (1988). Problemas aritméticos. Madrid: Síntesis.

Ramírez, R. (2012). Habilidades de visualización de los alumnos con talento matemático. Tesis Doctoral. Universidad de Granada.

Silver, E. (1994). On Mathematical problem posing. For the Learning of Mathematics, 14(1), 19-28.

Silver, E. \& Cai, J. (1996). An analysis of arithmetic problem posing by middle school students. Journal for Research in Mathematics Education, 27(5), 521-539. doi:10.2307/749846

Silver, E. \& Cai, J. (2005). Assessing students' mathematical problem posing. Teaching Children Mathematics, 12(3), 129-135.

Stoyanova, E. (1998). Problem posing in mathematics classrooms. En A. McIntosh y N. Ellerton (Eds.), Research in Mathematics Education: a contemporary perspective. (pp. 164-185). Edit Cowan University: MASTEC.

Villarraga, M., Martínez, P. \& Benavides, M. (2004). Hacia la definición del término talento. En M. Benavides, A. Maz, E. Castro y R. Blanco (Eds), La educación de niños con talento en Iberoamérica (pp. 25-35). Santiago (Chile): OREALC/Unesco. 